\title{
On isometric embeddings of Wasserstein spaces - the discrete case
}

Article

Accepted Version

Creative Commons: Attribution-Noncommercial-No Derivative Works 4.0

Geher, G. P., Titkos, T. and Virosztek, D. (2019) On isometric embeddings of Wasserstein spaces - the discrete case. Journal of Mathematical Analysis and Applications, 480 (2). 123435. ISSN 0022-247X doi:

https://doi.org/10.1016/j.jmaa.2019.123435 Available at https://centaur.reading.ac.uk/85683/

It is advisable to refer to the publisher's version if you intend to cite from the work. See Guidance on citing.

To link to this article DOI: http://dx.doi.org/10.1016/j.jmaa.2019.123435

Publisher: Elsevier

All outputs in CentAUR are protected by Intellectual Property Rights law, including copyright law. Copyright and IPR is retained by the creators or other copyright holders. Terms and conditions for use of this material are defined in the End User Agreement.

\section{www.reading.ac.uk/centaur}

\section{CentAUR}

Central Archive at the University of Reading 
Reading's research outputs online 


\title{
ON ISOMETRIC EMBEDDINGS OF WASSERSTEIN SPACES - THE DISCRETE CASE
}

\author{
GYÖRGY PÁL GEHÉR, TAMÁS TITKOS, AND DÁNIEL VIROSZTEK
}

\begin{abstract}
The aim of this short paper is to offer a complete characterization of all (not necessarily surjective) isometric embeddings of the Wasserstein space $\mathcal{W}_{p}(\mathcal{X})$, where $\mathcal{X}$ is a countable discrete metric space and $0<p<\infty$ is any parameter value. Roughly speaking, we will prove that any isometric embedding can be described by a special kind of $\mathcal{X} \times(0,1]$ indexed family of nonnegative finite measures. Our result implies that a typical non-surjective isometric embedding of $\mathcal{W}_{p}(\mathcal{X})$ splits mass and does not preserve the shape of measures. In order to stress that the lack of surjectivity is what makes things challenging, we will prove alternatively that $\mathcal{W}_{p}(\mathcal{X})$ is isometrically rigid for all $0<p<\infty$.
\end{abstract}

\section{INTRODUCTION}

In order to understand a mathematical structure, it is natural to start with exploring its symmetries. In the case of metric spaces, this leads naturally to the study of isometries, and more generally, to the study of isometric embeddings. As there is a crucial difference between these two types of maps, which is very important to emphasize for our main result, let us fix their definitions here:

- an isometry (sometimes called surjective isometry) is a bijective map that preserves the distance between elements;

- an isometric embedding is a (not necessarily surjective) map that preserves the distance.

Of course, every isometry is an isometric embedding, but usually there are several isometric embeddings that are non-surjective, hence not isometries. The investigation of isometries has a long history, let us first introduce some of it, in particular those results which are most relevant to this paper. One of the pioneers of these investigations was Banach, who presented the first characterizations of isometries on several classical spaces. Probably the most famous one is the Banach-Stone theorem, which says that isometries between $C(K)$-type spaces are induced by homeomorphisms of the underlying spaces followed by possible changes of sign in the function values on clopen (i.e., both closed and open) sets. When speaking only about this particular theorem, there is a vast literature of interesting generalizations (for an elegant non-commutative version see [10]). We mention briefly here only those in which metric spaces of probability measures are involved (for a more detailed survey we refer the reader to [20]). The first result in this direction is the description of all isometries of the non-linear space of all Borel probability measures over $\mathbb{R}$ with respect to the Kolmogorov-Smirnov distance,

2010 Mathematics Subject Classification. Primary: 54E40; 46E27 Secondary: 60A10; 60B05.

Key words and phrases. Wasserstein space, isometric embeddings, probability measures, discrete metric.

Gehér was supported by the Leverhulme Trust Early Career Fellowship (ECF-2018-125), and partially by the Hungarian National Research, Development and Innovation Office - NKFIH (K115383); Titkos was supported by the Hungarian National Research, Development and Innovation Office - NKFIH (PD128374), and by the János Bolyai Research Scholarship of the Hungarian Academy of Sciences; Virosztek was supported by the ISTFELLOW program of the Institute of Science and Technology Austria (project code IC1027FELL01), and partially by the Hungarian National Research, Development and Innovation Office - NKFIH (K124152). 
namely, Dolinar and Molnár showed in [4] that they are always induced by homeomorphisms of $\mathbb{R}$ (see also [14]). Another metric appearing in statistics is the Kuiper metric, which was discussed recently in [7] in full detail. Maybe the most relevant metrics in probability theory are those that metrise the weak converge like the Lévy-, the Lévy-Prokhorov-, and the Wasserstein metrics. Molnár proved in [15] that the space of all Borel probability measures over $\mathbb{R}$ endowed with the Lévy metric is isometrically rigid, i.e. every isometry is induced by an isometry of the underlying space $\mathbb{R}$. Generalizing Molnár's result, it has been proved in [8] that the space of all of Borel probability measures over a real and separable Banach space endowed with the Lévy-Prokhorov metric is also isometrically rigid.

The most important developments in our considerations have been done by Bertrand and Kloeckner in connection with the Wasserstein metric [2, 3, 11]. Besides that the Wasserstein distance metrises the weak convergence of probability measures, its importance also lies in its role in geometric investigations of metric spaces (for more information see [13, 16, 17, 18, 19] and the references therein). Among many other deep results, Bertrand and Kloeckner have managed to prove that the Wasserstein space $\mathcal{W}_{2}(X)$ is isometrically rigid for any negatively curved geodesically complete Hadamard space $X$. We highlight also Kloeckner's results on the isometry group of $\mathcal{W}_{2}\left(\mathbb{R}^{n}\right)$, in particular, isometries of $\mathcal{W}_{2}\left(\mathbb{R}^{n}\right)$ are usually not induced by only one mapping of $\mathbb{R}^{n}$ - unlike in the aforementioned results. Moreover, it turned out (which is one of the main results of [11]) that $\mathcal{W}_{2}(\mathbb{R})$ admits exotic isometries that does not even preserve the shape of measures. This result is pretty uncommon and it raises several questions (see Section 8 in [11], and also the last section of this paper). Moreover, the exotic isometry flow appearing in the description of $\operatorname{Isom}\left(\mathcal{W}_{2}(\mathbb{R})\right.$ ) (see Section 5 in [11]) is not very explicit, although constructive. One can imagine that things could become even more complicated when one drops the surjectivity condition and tries to characterize general isometric embeddings.

Concerning the characterization of isometric embeddings - which is our main interest in this paper - there are very few results in the literature. The main reason is that usually there is no hope for finding an elegant description of all isometric embeddings, because very wild maps can satisfy the distance preserving property. However, there are a few cases when the characterization of all isometric embeddings is known due to the underlying additional structure of the metric space - let us now mention two of them. First, it is well-known that all isometric embeddings of strictly convex real Banach spaces are automatically affine (i.e. linear up to translation), so in order to characterize their isometric embeddings it is enough to explore the structure of their linear norm-preserving operators. The latter was done by Lamperti in [12] for $L^{p}$ spaces over $\sigma$-finite measure spaces when $1<p<\infty, p \neq 2$. Therefore a characterization of isometric embeddings of these $L^{p}$ spaces follows from Lamperti's result. Another example is the famous Wigner's theorem about quantum mechanical symmetry transformations, which gives a characterization of isometric embeddings of the space of all rank-one projections of a Hilbert space with respect to the operator norm, see e.g. [5] (see also [6] for a generalization). In this case there is a certain projective structure which can be taken advantage of.

Our aim in this paper is to offer a complete description of all isometric embeddings of the Wasserstein space $\mathcal{W}_{p}(\mathcal{X})$, where $\mathcal{X}$ is a countable discrete metric space and $0<p<\infty$ is any parameter. Roughly speaking, we will prove that any isometric embedding can be described by a special kind of $\mathcal{X} \times(0,1]$-indexed family of nonnegative finite measures, and vice versa, any such family of measures determines a distance preserving transformation. In the study of isometries it is a quite usual phenomenon that isometries also preserve some other underlying structure of the metric space. For instance, in [2, 4, 7, 8] and [15] it turned out - as a 
consequence of the main theorems - that every isometry is automatically affine, i.e. preserves convex combinations of measures; and in [11] that every isometry of $\mathcal{W}_{2}(\mathbb{R})$ preserves geodesically convex combinations of measures. Here we shall see that for a typical non-surjective isometric embedding of $\mathcal{W}_{p}(\mathcal{X})$ this is not true anymore. Moreover, we will also see that a general isometric embedding splits mass and does not preserve the shape of measures. To demonstrate that the real difficulty here is the lack of surjectivity, we will also prove alternatively that $\mathcal{W}_{p}(\mathcal{X})$ is isometrically rigid for all $0<p<\infty$. It can be somewhat surprising that although the $W_{\infty}$-distance can be obtained as a limit of $W_{p}$ distances, the structure of its isometric embeddings (and its isometry group) is completely different.

After this brief introductory part, we continue by fixing the setting, and introducing the necessary definitions and notations. Let $X \neq \emptyset$ be a countable set, and let $\rho: X^{2} \rightarrow\{0,1\}$ be the discrete metric, i.e., $\rho(x, y):=1$ if $x \neq y$ and $\rho(x, x):=0$ for all $x, y \in X$. To avoid trivialities, we will always assume that $X$ has at least two elements. We will denote the Polish space $(X, \rho)$ shortly by $\mathcal{X}$. The symbols $\mathcal{P}(\mathcal{X})$ and $\mathcal{M}(\mathcal{X})$ stand for the sets of probability measures and nonnegative finite measures on the power set (i.e., the Borel $\sigma$-algebra) of $\mathcal{X}$, respectively. For $\mu \in \mathcal{M}(\mathcal{X})$ and $T \subseteq \mathcal{X}$ the symbol $\left.\mu\right|_{T} \in \mathcal{M}(\mathcal{X})$ stands for the restricted measure defined by

$$
\left.\mu\right|_{T}(A):=\mu(A \cap T) \quad \text { for all } A \subseteq \mathcal{X} .
$$

As usual, $\delta_{x}$ denotes the Dirac measure (or point mass) concentrated to $x \in \mathcal{X}$, i.e. $\delta_{x}(A)=1$ if $x \in A$, and $\delta_{x}(A)=0$ if $x \notin A$. The set of Dirac measures will be denoted by $\Delta(\mathcal{X})=$ $\left\{\delta_{x} \mid x \in \mathcal{X}\right\}$. It is obvious that each element of $\mathcal{M}(\mathcal{X})$ can be written as a weighted sum of Dirac measures, namely $\mu=\sum_{x \in X} \mu(\{x\}) \cdot \delta_{x}$. The support of $\mu$ in this special case is just the set $S_{\mu}=\{x \in \mathcal{X} \mid \mu(\{x\})>0\}$. As it is known (see Theorem 36.1 in [1]), the set of nonnegative finite measures on a fixed $\sigma$-algebra is a lattice. In this discrete case, the greatest lower bound $\mu \wedge \nu$ of two measures $\mu, \nu \in \mathcal{M}(\mathcal{X})$ can be calculated easily as

$$
\mu \wedge \nu=\sum_{x \in \mathcal{X}} \min \{\mu(\{x\}), \nu(\{x\})\} \cdot \delta_{x} .
$$

In order to introduce the Wasserstein space $\mathcal{W}_{p}(\mathcal{X})$, we need some more definitions. Since

$$
\mathcal{W}_{p}(\mathcal{X}):=\left\{\mu \in \mathcal{P}(\mathcal{X}) \mid \int_{\mathcal{X}} \rho\left(x, x_{0}\right)^{p} d \mu(x)<\infty \quad \text { for some (and hence all) } x_{0} \in \mathcal{X}\right\}
$$

coincides with $\mathcal{P}(\mathcal{X})$ and $\rho^{p}=\rho$ for all parameter values $p \in(0, \infty)$ we will write always $\mathcal{P}(\mathcal{X})$ without indicating $p$. We say that a Borel probability measure $\pi$ on $\mathcal{X}^{2}$ is a coupling of $\mu, \nu \in \mathcal{P}(\mathcal{X})$ (and we write shortly $\pi \in \mathcal{C}(\mu, \nu)$ ), if it satisfies

$$
\pi(A \times \mathcal{X})=\mu(A) \quad \text { and } \quad \pi(\mathcal{X} \times A)=\nu(A) \quad \text { for all } A \subseteq \mathcal{X} .
$$

The $p$-Wasserstein distance (which is indeed a metric on $\mathcal{P}(\mathcal{X})$ ) of $\mu$ and $\nu$ is defined by

$$
W_{p}(\mu, \nu):=\left(\inf _{\pi \in \mathcal{C}(\mu, \nu)} \int_{\mathcal{X}^{2}} \rho(x, y)^{p} d \pi(x, y)\right)^{1 / p}
$$

if $p \geq 1$, and by

$$
W_{p}(\mu, \nu):=\inf _{\pi \in \mathcal{C}(\mu, \nu)} \int_{\mathcal{X}^{2}} \rho(x, y)^{p} d \pi(x, y)
$$


if $p \in(0,1)$. For more details and historical comments we refer the reader to [18, Chapter 6]. Observe that $W_{p}(\mu, \nu)=W_{1}(\mu, \nu)$ holds for all $\mu, \nu \in \mathcal{P}(\mathcal{X})$ and $p \in(0,1)$, because $\rho^{p}=\rho$. The metric space $\left(\mathcal{P}(\mathcal{X}), W_{p}\right)$ is denoted shortly by $\mathcal{W}_{p}(\mathcal{X})$ and is called the $p$-Wasserstein space over $\mathcal{X}$. We will call a transformation $f: \mathcal{W}_{p}(\mathcal{X}) \rightarrow \mathcal{W}_{p}(\mathcal{X})$ an isometric embedding if

$$
W_{p}(\mu, \nu)=W_{p}(f(\mu), f(\nu)) \quad \text { for all } \mu, \nu \in \mathcal{W}_{p}(\mathcal{X}) .
$$

In the next section we present our main theorem, which is a complete characterization of isometric embeddings of $\mathcal{W}_{p}(\mathcal{X})$.

\section{Characterization OF ISOMETRIC EMBEDDingS}

First we, express the Wasserstein distance in terms of the greatest lower bound of measures. Note that this formula might be folklore, however, for the sake of completeness and for the convenience of the reader, we include it.

Proposition. For any $1 \leq p<\infty$ and $\mu, \nu \in \mathcal{W}_{p}(\mathcal{X})$ we have

$$
W_{p}^{p}(\mu, \nu)=1-(\mu \wedge \nu)(\mathcal{X}) .
$$

Proof. The formula (4) is obviously true for $\mu=\nu$, hence we assume in the sequel that $\mu \neq \nu$. For any coupling measure $\pi \in \mathcal{C}(\mu, \nu)$ and point $x \in \mathcal{X}$ we notice that

$$
\pi(\{(x, x)\}) \leq \min \{\pi(\{x\} \times \mathcal{X}), \pi(\mathcal{X} \times\{x\})\}=\min \{\mu(\{x\}), \nu(\{x\})\},
$$

thus for the diagonal $\operatorname{Diag}\left(\mathcal{X}^{2}\right)=\{(x, x) \mid x \in \mathcal{X}\}$ we conclude $\pi\left(\operatorname{Diag}\left(\mathcal{X}^{2}\right)\right) \leq(\mu \wedge \nu)(\mathcal{X})$. According to (2) this inequality implies

$$
W_{p}^{p}(\mu, \nu)=\inf _{\pi \in \mathcal{C}(\mu, \nu)} \int_{\mathcal{X}^{2} \backslash \operatorname{Diag}\left(\mathcal{X}^{2}\right)} 1 d \pi(x, y)=\inf _{\pi \in \mathcal{C}(\mu, \nu)}\left(1-\pi\left(\operatorname{Diag}\left(\mathcal{X}^{2}\right)\right)\right) \geq 1-(\mu \wedge \nu)(\mathcal{X}),
$$

therefore it is enough to construct a coupling $\pi^{*} \in \mathcal{C}(\mu, \nu)$ for which $\pi^{*}\left(\operatorname{Diag}\left(\mathcal{X}^{2}\right)\right)=(\mu \wedge \nu)(\mathcal{X})$ holds. To this aim, let us introduce some notation. For a signed measure $\rho$ on $\mathcal{X}$, let $\rho_{+}$denote the nonnegative measure given by $\rho_{+}(\{x\})=\max \{0, \rho(\{x\})\}$ for every $x \in \mathcal{X}$. (In other words, $\rho_{+}$is the nonnegative part of $\rho$.) Furthermore, for $\eta, \xi \in \mathcal{M}(\mathcal{X})$, we denote by $\eta \otimes \xi$ the product measure of $\eta$ and $\xi$, that is, $(\eta \otimes \xi)(A \times B)=\eta(A) \cdot \xi(B)$ for all $A, B \subseteq \mathcal{X}$. Finally, let $i: \mathcal{X} \rightarrow \mathcal{X}^{2} ; x \mapsto(x, x)$ be the diagonal embedding of $\mathcal{X}$ into $\mathcal{X}^{2}$, and let us define $\pi^{*}$ by

$$
\pi^{*}:=i_{\#}(\mu \wedge \nu)+\frac{(\mu-\nu)_{+} \otimes(\nu-\mu)_{+}}{1-(\mu \wedge \nu)(\mathcal{X})}
$$

where $i_{\#}(\mu \wedge \nu)$ is the push-forward measure of $\mu \wedge \nu$ by the map $i$, that is,

$$
\left(i_{\#}(\mu \wedge \nu)\right)(A)=(\mu \wedge \nu)\left(i^{-1}(A)\right) \text { for all Borel sets } A \subseteq \mathcal{X}^{2} .
$$

Note that $(\mu-\nu)_{+}(\mathcal{X})=(\nu-\mu)_{+}(\mathcal{X})=1-(\mu \wedge \nu)(\mathcal{X})$, hence $\pi^{*}$ is indeed a probability measure on $\mathcal{X}^{2}$. Moreover, it is also clear that $\pi^{*}\left(\operatorname{Diag}\left(\mathcal{X}^{2}\right)\right)=(\mu \wedge \nu)(\mathcal{X})$. To see that $\pi^{*}$ is a coupling of $\mu$ and $\nu$, we first check that $\pi^{*}(A \times \mathcal{X})=\mu(A)$ holds for every $A \subseteq \mathcal{X}$ by the following simple computation:

$\pi^{*}(A \times \mathcal{X})=(\mu \wedge \nu)(A)+(\mu-\nu)_{+}(A) \cdot \frac{(\nu-\mu)_{+}(\mathcal{X})}{1-(\mu \wedge \nu)(\mathcal{X})}=(\mu \wedge \nu)(A)+(\mu-\nu)_{+}(A)=\mu(A)$.

As identity $\pi^{*}(\mathcal{X} \times A)=\nu(A)$ can be verified similarly, we conclude (4) as it was claimed. 
Note that for $0<p<1$ we have $W_{p}(\mu, \nu)=W_{1}(\mu, \nu)=1-(\mu \wedge \nu)(\mathcal{X})$. As one consequence of (4) we can easily show that the Wasserstein space $\mathcal{W}_{p}(\mathcal{X})$ is isometrically rigid. That is, the isometry group of $\mathcal{W}_{p}(\mathcal{X})$ (consisting of all bijective distance-preserving transformations of $\mathcal{W}_{p}(\mathcal{X})$ ) is isomorphic to the isometry group of $\mathcal{X}$, which is just the permutation group of $\mathcal{X}$.

Corollary. Let $p \in(0, \infty)$ and $f: \mathcal{W}_{p}(\mathcal{X}) \rightarrow \mathcal{W}_{p}(\mathcal{X})$ be an isometry, that is an isometric embedding which is surjective. Then there exists a bijection $\sigma: \mathcal{X} \rightarrow \mathcal{X}$ such that $f$ is a push-forward of $\sigma$, i.e.

$$
f(\mu)(\{x\})=\mu\left(\left\{\sigma^{-1}(x)\right\}\right) \quad \text { for all } x \in \mathcal{X} .
$$

Conversely, if a bijection $\sigma: \mathcal{X} \rightarrow \mathcal{X}$ is given, then (5) defines an isometry.

Proof. By (4) we have

$$
S_{\mu} \cap S_{\nu}=\emptyset \Longleftrightarrow W_{p}^{p}(\mu, \nu)=1 \quad \text { for all } \mu, \nu \in \mathcal{W}_{p}(\mathcal{X}) .
$$

Since $f$ is a bijection, the restriction $\left.f\right|_{\Delta(\mathcal{X})}$ is a bijection of $\Delta(\mathcal{X})$. Indeed, assume that $x_{1}, x_{2} \in S_{f\left(\delta_{x}\right)}, x_{1} \neq x_{2}$ happens, then we have $W_{p}^{p}\left(f\left(\delta_{x}\right), \delta_{x_{i}}\right) \neq 1(i \in\{1,2\})$, therefore taking the inverse images gives

$$
W_{p}^{p}\left(\delta_{x}, f^{-1}\left(\delta_{x_{1}}\right)\right) \neq 1, \quad W_{p}^{p}\left(\delta_{x}, f^{-1}\left(\delta_{x_{2}}\right)\right) \neq 1, \quad W_{p}^{p}\left(f^{-1}\left(\delta_{x_{1}}\right), f^{-1}\left(\delta_{x_{2}}\right)\right)=1,
$$

which is a contradiction by (6). Hence the $f$-image of every Dirac mass is a Dirac mass, and we argue similarly for the inverse $f^{-1}$.

Therefore the action of $f$ on $\Delta(\mathcal{X})$ induces a bijection $\sigma$ on $\mathcal{X}$ defined by $f\left(\delta_{x}\right)=\delta_{\sigma(x)}$. Now, take any measure $\mu \in \mathcal{P}(\mathcal{X})$ and any $x \in \mathcal{X}$, and observe by (4) that

$$
\mu\left(\left\{\sigma^{-1}(x)\right\}\right)=\left(\mu \wedge \delta_{\sigma^{-1}(x)}\right)(\mathcal{X})=\left(f(\mu) \wedge f\left(\delta_{\sigma^{-1}(x)}\right)\right)(\mathcal{X})=\left(f(\mu) \wedge \delta_{x}\right)(\mathcal{X})=f(\mu)(\{x\}),
$$

which proves one direction. The reverse direction follows from the fact that the distance is invariant under permutations of the underlying space.

Now, we turn to the much more interesting problem of characterizing all isometric embeddings of $\mathcal{W}_{p}(\mathcal{X})$. In order to demonstrate that indeed quite wild maps can preserve the Wasserstein distance, we begin with two examples of isometric embeddings which are not affine, moreover, they split Dirac masses and therefore do not preserve the shape of measures.

Example 1. Let us fix a parameter value $p \in(0, \infty)$, and let $\mathcal{X}=\{1,2,3, \ldots\}$ be the set of all natural numbers endowed with the discrete metric. Define $f: \mathcal{W}_{p}(\mathcal{X}) \rightarrow \mathcal{W}_{p}(\mathcal{X})$ as

$$
f\left(\sum_{n \in \mathcal{X}} c_{n} \cdot \delta_{n}\right)=\sum_{n \in \mathcal{X}}\left[\ln \left(1+c_{n}\right) \cdot \delta_{2 n}+\left(c_{n}-\ln \left(1+c_{n}\right)\right) \cdot \delta_{2 n+1}\right] .
$$

One can show by definition and using (4) that this is indeed a non-surjective isometric embedding. Observe that the range of $f$ does not contain any Dirac mass. What happens here is roughly speaking the following: $f$ splits Dirac masses

$$
f\left(\delta_{n}\right)=\ln 2 \cdot \delta_{2 n}+(1-\ln 2) \cdot \delta_{2 n+1},
$$

and redistributes weights. On one hand, if $\mathrm{f} n \neq m$, then $S_{f\left(\delta_{n}\right)} \cap S_{f\left(\delta_{m}\right)}=\emptyset$, thus $f$ induces a partition of $\mathcal{X}$, in fact, the support of $f(\mu)$ is always the disjoint union

$$
S_{f(\mu)}=\bigcup_{n \in S_{\mu}} S_{f\left(\delta_{n}\right)}=\bigcup_{n \in S_{\mu}}\{2 n, 2 n+1\} .
$$


On the other hand, we see that if $\mu(\{n\})=c_{n}$, then $f(\mu)(\{2 n, 2 n+1\})=c_{n}$, and if $\mu(\{n\}) \leq$ $\nu(\{n\})$ then

$$
\left.f(\mu)\right|_{\{2 n, 2 n+1\}} \leq\left. f(\nu)\right|_{\{2 n, 2 n+1\}} .
$$

The second example is even wilder.

Example 2. Let $\mathcal{X}$ be again the set of all natural numbers endowed with the discrete metric. Also let $p_{n}$ denote the $n$th prime number. Define $f: \mathcal{W}_{p}(\mathcal{X}) \rightarrow \mathcal{W}_{p}(\mathcal{X})$ as

$$
f\left(\sum_{n \in \mathcal{X}} c_{n} \cdot \delta_{n}\right)=\sum_{n \in \mathcal{X}}\left(\sum_{j=1}^{N\left(c_{n}\right)} \frac{1}{2^{j}} \delta_{p_{n}^{j}}+\left(c_{n}-\sum_{j=1}^{N\left(c_{n}\right)} \frac{1}{2^{j}}\right) \delta_{p_{n}^{N\left(c_{n}\right)+1}}\right),
$$

where for every $c \in[0,1)$ the non-negative integer $N(c)$ is defined by

$$
\sum_{j=1}^{N(c)} \frac{1}{2^{j}} \leq c<\sum_{j=1}^{N(c)+1} \frac{1}{2^{j}}
$$

and $N(1)=\infty$. Note that this map also shares similar properties mentioned in the previous example, which we do not repeat here. However, this map has a quite peculiar property, namely that every Dirac mass is mapped into an infinitely supported measure, but any other finitely supported measure is mapped into a finitely supported one. In particular, instead of (7) in general we only have

$$
S_{f(\mu)} \subset \bigcup_{n \in S_{\mu}} S_{f\left(\delta_{n}\right)}=\bigcup_{n \in S_{\mu}}\left\{p_{n}^{j}: j=1,2, \ldots\right\}
$$

Now, we are in the position to claim and prove our main result. We will see that in a particular sense every isometric embedding $f$ looks like the above examples: $f$ will induce a partition on $\mathcal{X}$ and a family of nonnegative finite measures satisfying some special properties from which we can construct the whole action of $f$.

Theorem. Let $p \in(0, \infty)$ and $f: \mathcal{W}_{p}(\mathcal{X}) \rightarrow \mathcal{W}_{p}(\mathcal{X})$ be an isometric embedding, i.e.,

$$
W_{p}(\mu, \nu)=W_{p}(f(\mu), f(\nu)) \quad \text { for all } \quad \mu, \nu \in \mathcal{W}_{p}(\mathcal{X}) .
$$

Then there exists a unique family of measures

$$
\Phi:=\left(\varphi_{x, t}\right)_{x \in \mathcal{X}, t \in(0,1]} \in \mathcal{M}(\mathcal{X})^{\mathcal{X} \times(0,1]}
$$

that satisfies the properties

(a) for all $x \neq y: S_{\varphi_{x, 1}} \cap S_{\varphi_{y, 1}}=\emptyset$,

(b) for all $x \in \mathcal{X}$ and $0<t \leq 1: \varphi_{x, t}(\mathcal{X})=t$,

(c) for all $x \in \mathcal{X}$ and $0<s<t \leq 1: \varphi_{x, s} \leq \varphi_{x, t}$,

and that generates $f$ in the following sense

$$
f(\mu)=\sum_{x \in S_{\mu}} \varphi_{x, \mu(\{x\})} \quad \text { for all } \quad \mu \in \mathcal{W}_{p}(\mathcal{X})
$$

Conversely, every $\mathcal{X} \times(0,1]$-indexed family of measures satisfying (a)-(c) generates an isometric embedding via the formula 10 . 
Proof. As $\mathcal{W}_{p}(\mathcal{X})$ spaces are indistinguishable from $\mathcal{W}_{1}(\mathcal{X})$ whenever $p \in(0,1)$, we will always assume for the sake of simplicity that $p \geq 1$. Let $f$ be a fixed isometric embedding, our first step is to construct the family $\Phi$. Observe that by (4) we have

$$
(f(\mu) \wedge f(\nu))(\mathcal{X})=(\mu \wedge \nu)(\mathcal{X}) \quad \text { for all } \mu, \nu \in \mathcal{P}(\mathcal{X})
$$

in particular, $f$ preserves the disjointness of supports in both directions:

$$
S_{\mu} \cap S_{\nu}=\emptyset \quad \Longleftrightarrow \quad S_{f(\mu)} \cap S_{f(\nu)}=\emptyset \quad \text { for all } \mu, \nu \in \mathcal{P}(\mathcal{X}) .
$$

As a special case we obtain $S_{f\left(\delta_{x}\right)} \cap S_{f\left(\delta_{y}\right)}=\emptyset$ whenever $x \neq y$. Since every $\mu \in \mathcal{P}(\mathcal{X})$ can be written in the form $\mu=\sum_{x \in \mathcal{X}} \mu(\{x\}) \cdot \delta_{x}$, and the sets $S_{f\left(\delta_{x}\right)}(x \in \mathcal{X})$ are pairwise disjoint, it is a natural idea to define a map $F: \Delta(\mathcal{X})_{\leq} \rightarrow \mathcal{M}(\mathcal{X})$ on $\Delta(\mathcal{X})_{\leq}:=\left\{t \cdot \delta_{x} \mid t \in(0,1], x \in \mathcal{X}\right\}$ for which $\left.F\right|_{\Delta(\mathcal{X})}=\left.f\right|_{\Delta(\mathcal{X})}$ holds, and which is compatible with $f$. By compatibility we mean that if $0<t=\mu(\{x\})=\nu(\{x\})$, then

$$
\left.f(\mu)\right|_{S_{f\left(\delta_{x}\right)}}=\left.f(\nu)\right|_{S_{f\left(\delta_{x}\right)}}=F\left(t \cdot \delta_{x}\right)
$$

is satisfied. Of course it is not clear at this point why $F$ should be well-defined. In order to see that, it is enough to prove that if $0<s=\mu(\{x\}) \leq \nu(\{x\})=t$ holds for $\mu, \nu \in \mathcal{P}(\mathcal{X})$, then $\left.f(\mu)\right|_{S_{f\left(\delta_{x}\right)}} \leq\left. f(\nu)\right|_{S_{f\left(\delta_{x}\right)}}$.

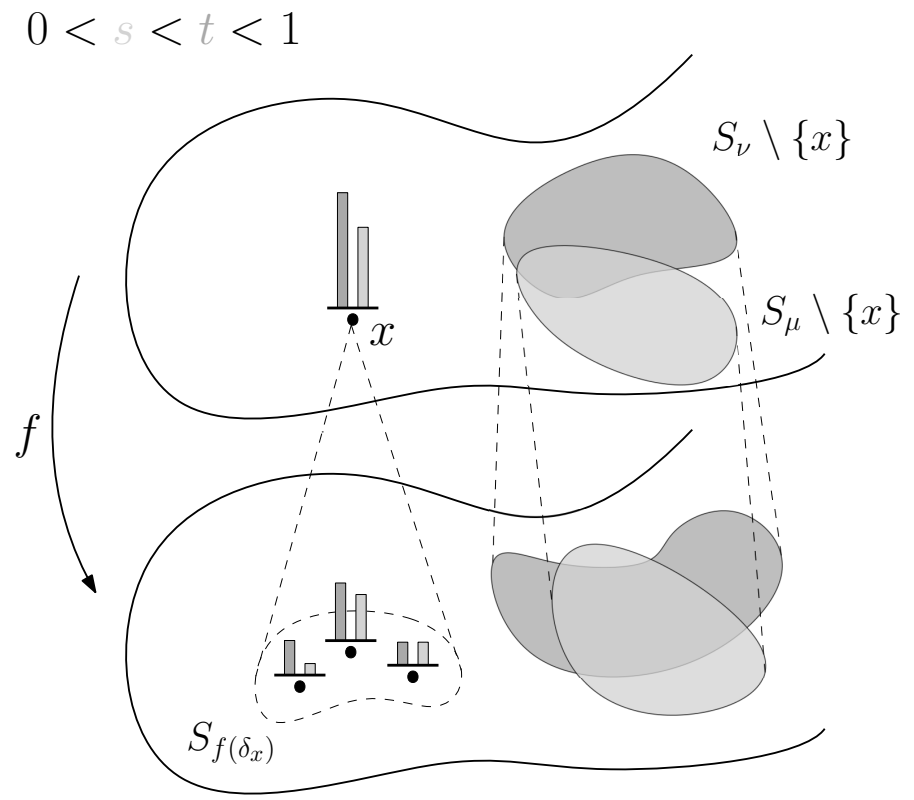

FIGURE 1. $\left.f(\mu)\right|_{S_{f\left(\delta_{x}\right)}} \leq\left. f(\nu)\right|_{S_{f\left(\delta_{x}\right)}}$.

We start by understanding the structure of $f(\mu)$. First observe that by (11)

$$
\mu(\{x\})=\left(\delta_{x} \wedge \mu\right)(\mathcal{X})=\left(f\left(\delta_{x}\right) \wedge f(\mu)\right)(\mathcal{X})=\left(f\left(\delta_{x}\right) \wedge f(\mu)\right)\left(S_{f\left(\delta_{x}\right)}\right) \leq f(\mu)\left(S_{f\left(\delta_{x}\right)}\right)
$$

holds for all $x \in \mathcal{X}$. Since the supports of $f\left(\delta_{x}\right)$ are pairwise disjoint, we easily obtain that

$$
\mu(\{x\})=f(\mu)\left(S_{f\left(\delta_{x}\right)}\right)=\left.f(\mu)\right|_{S_{f\left(\delta_{x}\right)}}(\mathcal{X}) \text { for all } x \in \mathcal{X}
$$


and hence that $S_{f(\mu)} \subseteq \bigcup_{x \in S_{\mu}} S_{f\left(\delta_{x}\right)}$. Now, for arbitrary probability measures $\mu$ and $\nu \in \mathcal{P}(\mathcal{X})$ we calculate

$$
\begin{aligned}
\sum_{x \in S_{\mu} \cap S_{\nu}} \min \{\mu(\{x\}), \nu(\{x\})\} & =(\mu \wedge \nu)(\mathcal{X})=(f(\mu) \wedge f(\nu))(\mathcal{X}) \\
& =\left[\left(\left.\sum_{x \in S_{\mu}} f(\mu)\right|_{S_{f\left(\delta_{x}\right)}}\right) \wedge\left(\left.\sum_{x \in S_{\nu}} f(\nu)\right|_{S_{f\left(\delta_{x}\right)}}\right)\right](\mathcal{X}) \\
& =\sum_{x \in S_{\mu} \cap S_{\nu}}\left(\left.\left.f(\mu)\right|_{S_{f\left(\delta_{x}\right)}} \wedge f(\nu)\right|_{S_{f\left(\delta_{x}\right)}}\right)(\mathcal{X}) \\
& \leq \sum_{x \in S_{\mu} \cap S_{\nu}} \min \left\{\left(\left.f(\mu)\right|_{S_{f\left(\delta_{x}\right)}}(\mathcal{X}),\left.f(\nu)\right|_{S_{f\left(\delta_{x}\right)}}(\mathcal{X})\right\}\right. \\
& =\sum_{x \in S_{\mu} \cap S_{\nu}} \min \{\mu(\{x\}), \nu(\{x\})\}
\end{aligned}
$$

which forces the following for all $x \in S_{\mu} \cap S_{\nu}$ :

$$
\left(\left.\left.f(\mu)\right|_{S_{f\left(\delta_{x}\right)}} \wedge f(\nu)\right|_{S_{f\left(\delta_{x}\right)}}\right)(\mathcal{X})=\min \left\{\left(\left.f(\mu)\right|_{S_{f\left(\delta_{x}\right)}}(\mathcal{X}),\left.f(\nu)\right|_{S_{f\left(\delta_{x}\right)}}(\mathcal{X})\right\} .\right.
$$

At this point let us note the trivial fact that the partial ordering on the set of all non-negative finite measures can be expressed by the measure of $\mathcal{X}$. Indeed, we have

$$
\alpha \leq \beta \quad \Longleftrightarrow \quad \alpha(\mathcal{X}) \leq(\alpha \wedge \beta)(\mathcal{X}) \quad \text { for all } \alpha, \beta \in \mathcal{M}(\mathcal{X})
$$

Therefore, if $0<s=\mu(\{x\}) \leq \nu(\{x\})=t$ holds for a point $x \in S_{\mu} \cap S_{\nu}$, then by (14) and (15) we obtain

as was claimed.

$$
\left.f(\mu)\right|_{S_{f\left(\delta_{x}\right)}} \leq\left. f(\nu)\right|_{S_{f\left(\delta_{x}\right)}}
$$

Now, we define the family $\Phi$ by

$$
\varphi_{x, t}:=F\left(t \cdot \delta_{x}\right) \quad(x, t) \in \mathcal{X} \times(0,1] .
$$

From here (a)-(c) is obvious, and hence 10$)$ is also straightforward.

Finally, let us prove the reverse statement. Assume that $\Phi=\left(\varphi_{x, t}\right)_{x \in \mathcal{X}, t \in(0,1]} \in \mathcal{M}(\mathcal{X})^{\mathcal{X} \times(0,1]}$ is a family satisfying (a)-(c), then the following calculation shows that the map defined by (10) is indeed an isometric embedding:

$$
\begin{aligned}
& W_{p}^{p}(f(\mu), f(\nu))=1-[f(\mu) \wedge f(\nu)](\mathcal{X}) \\
& =1-\left[\left(\sum_{x \in S_{\mu}} \varphi_{x, \mu(\{x\})}\right) \wedge\left(\sum_{x \in S_{\nu}} \varphi_{x, \nu(\{x\})}\right)\right](\mathcal{X}) \\
& =1-\sum_{x \in S_{\mu} \cap S_{\nu}}\left[\varphi_{x, \mu(\{x\})} \wedge \varphi_{x, \nu(\{x\})}\right](\mathcal{X}) \\
& =1-\sum_{x \in S_{\mu} \cap S_{\nu}} \varphi_{x, \min }\{\mu(\{x\}), \nu(\{x\})\}(\mathcal{X}) \\
& =1-\sum_{x \in S_{\mu} \cap S_{\nu}} \min \{\mu(\{x\}), \nu(\{x\})\}=1-(\mu \wedge \nu)(\mathcal{X})=W_{p}^{p}(\mu, \nu) .
\end{aligned}
$$


Let us now make a few remarks on our main result. As we noted earlier, in the theory of isometries it is a quite typical phenomenon that isometries also preserve some other structure of the metric space. For instance, in [2, 4, 7, 8, 15] all isometries were induced by either an isometry or a homeomorphism of the underlying space (which were either $\mathbb{R}$, or a real separable Banach space, or a negatively curved geodesically complete Hadamard space). In particular, from these results it follows that isometries of those spaces are automatically affine, hence for any isometry $\phi$ once we know the $\phi$-images of two measures, the $\phi$-images of their convex combinations are also determined. Furthermore, it follows easily that the action of $\phi$ on Dirac masses determines its action on the whole metric space. However, this is definitely not the case for general isometric embeddings of $\mathcal{W}_{p}(\mathcal{X})$ (although it is the case for its isometries as we saw in Corollary). To demonstrate this, assume we know the $f$-images of two Dirac masses $\delta_{x}$ and $\delta_{y}, x \neq y$, and consider an arbitrary third measure supported on $\{x, y\}: \mu=c \cdot \delta_{x}+(1-c) \cdot \delta_{y} \in$ $\mathcal{P}(\mathcal{X}) \backslash\left\{\delta_{x}, \delta_{y}\right\}$. Then the only thing we can conclude for $f(\mu)$ is that it has the form $\mu_{x}+\mu_{y}$ where $\mu_{x} \leq f\left(\delta_{x}\right), \mu_{x}(\mathcal{X})=c$ and $\mu_{y} \leq f\left(\delta_{y}\right), \mu_{y}(\mathcal{X})=1-c$ (cf. the previous two examples). Moreover, it is not too hard to see that for any such choice of $\mu_{x}$ and $\mu_{y}$ we can construct an isometric embedding $f$ such that $f(\mu)=\mu_{x}+\mu_{y}$.

As was pointed out earlier, isometries of $W^{2}(\mathbb{R})$ automatically preserve geodesic convex combinations, however, an isometry of $W^{2}(\mathbb{R})$ is usually not determined by its action on Dirac masses, unlike in the previous paragraph. On the other hand, it is easy to see from the results of [11] that any isometry of $W^{2}(\mathbb{R})$ is determined by its action on the set $\Delta_{2}^{\prime}$ of all probability measures with two-point supports. It is interesting to note that any isometric embedding of $\mathcal{W}_{p}(\mathcal{X})$ is also determined by its action on the set $\Delta_{2}^{\prime}(\mathcal{X}):=\left\{\mu \in \mathcal{P}(\mathcal{X}): \# S_{\mu}=2\right\}$, although we have more flexibility than in the case of $W^{2}(\mathbb{R})$.

We also remark that if the underlying space $\mathcal{X}$ is finite, then isometric embeddings of $\mathcal{W}_{p}(\mathcal{X})$ are automatically surjective, hence they are implemented by a permutation of $\mathcal{X}$ according to Corollary.

Next, we would like to point out that the limiting case when $p=\infty$ is very different. Let $\mathcal{W}_{\infty}(\mathcal{X})$ be the set $\mathcal{P}(\mathcal{X})$ endowed with the metric $W_{\infty}(\mu, \nu)=\lim _{p \rightarrow \infty} W_{p}(\mu, \nu)$ (see Proposition 3 and the references in [9]). Then according to (4), we obtain that $W_{\infty}(\mu, \nu)=1$ if and only if $\mu \neq \nu$, thus $\mathcal{W}_{\infty}(\mathcal{X})$ is the set $\mathcal{P}(\mathcal{X})$ endowed with the discrete metric. Therefore any injective function $f: \mathcal{W}_{\infty}(\mathcal{X}) \rightarrow \mathcal{W}_{\infty}(\mathcal{X})$ is an isometric embedding, and any bijection is an isometry.

We close this paper with some remarks about Kloeckner's questions. First we recall that a map $f: \mathcal{W}_{p}(\mathcal{X}) \rightarrow \mathcal{W}_{p}(\mathcal{X})$ is called shape preserving if for all $\mu \in \mathcal{P}(\mathcal{X})$ there exists an isometry $\psi_{\mu}: \mathcal{X} \rightarrow \mathcal{X}$ (depending on $\mu$ ), such that $f(\mu)$ is the push-forward measure of $\mu$ by the map $\psi_{\mu}$. (Note that the isometries discussed in [2, 8, 15] are all shape preserving.) An isometry is called exotic if it is not shape preserving. (Note that the isometries discussed in [4, 7] are exotic in this particular sense, although each of them is induced by a transformation of $\mathbb{R}$, unlike in [11].) Now we are in the position to formulate Kloeckner's questions [11, Question 8.1 and Question 8.2]:

Q1 Is there a Polish (or Hadamard) space $\mathcal{X} \neq \mathbb{R}$ such that $\mathcal{W}_{2}(\mathcal{X})$ admits exotic isometries?

Q2 Is there a Polish space $\mathcal{X}$ whose Wasserstein space $\mathcal{W}_{2}(\mathcal{X})$ possesses an isometry that does not preserve the set of all Dirac masses?

We cannot answer exactly these questions because we are dealing with isometric embeddings, and not with bijections. However, according to our main result (also, it is worth to revisit the 
previous two examples), there are many isometric embeddings $f: \mathcal{W}_{2}(\mathcal{X}) \rightarrow \mathcal{W}_{2}(\mathcal{X})$ such that (i) $\mathrm{f}$ is exotic, and (ii) $f$ does not preserve the set of Dirac masses. Moreover, the parameter value $p=2$ is crucial as well, because as we have seen already, any bijection of $\mathcal{W}_{\infty}(\mathcal{X})$ is an isometry and thus both (i) and (ii) can happen even in the bijective case, if $p=\infty$.

Finally, concerning the above questions, let us remark that in a forthcoming paper we will discuss a Polish space $X$ such that for all $1<p<\infty$ the space $\mathcal{W}_{p}(X)$ is isometrically rigid, but the space $\mathcal{W}_{1}(X)$ has isometries that do not even preserve the set of all Dirac measures.

\section{REFERENCES}

[1] C.D. Aliprantis, and O. Burkinshaw, Principles of Real Analysis, Third Edition, Academic Press, San Diego, 1998.

[2] J. Bertrand, and B. Kloeckner, A geometric study of Wasserstein spaces: isometric rigidity in negative curvature, Int. Math. Res. Not. IMRN (2016), 1368-1386.

[3] J. Bertrand, and B. Kloeckner, A geometric study of Wasserstein spaces: Hadamard spaces, J. Topol. Anal. 4 (2012), 515-542.

[4] G. Dolinar, and L. Molnár, Isometries of the space of distribution functions with respect to the Kolmogorov-Smirnov metric, J. Math. Anal. Appl. 348 (2008), 494-498.

[5] Gy.P. Gehér, An elementary proof for the non-bijective version of Wigner's theorem, Phys. Lett. A 378 (2014), 2054-2057.

[6] Gy.P. Gehér, Wigner's theorem on Grassmann spaces, J. Funct. Anal. 273 (2017), 2994-3001.

[7] Gy.P. Gehér, Surjective Kuiper isometries, Houston J. Math. 44 (2018), 263-281.

[8] Gy.P. Gehér, and T. Titkos, A characterisation of isometries with respect to the Lévy-Prokhorov metric, Ann. Sc. Norm. Super. Pisa Cl. Sci. (5) (2018), in press.

[9] C. R. Givens, and R. M. Shortt, A class of Wasserstein metrics for probability distributions, Michigan Math. J. 31 (1984), 231-240.

[10] R.V. Kadison, Isometries of Operator Algebras, Ann. Of Math. (2) 54, (1951), 325-338.

[11] B. Kloeckner, A geometric study of Wasserstein spaces: Euclidean spaces, Ann. Sc. Norm. Super. Pisa Cl. Sci. (5) 9 (2010), 297-323.

[12] J. Lamperti, On the isometries of certain function-spaces, Pacific J. Math. 8 (1958), 459-466.

[13] J. Lott, and C. Villani, Ricci curvature for metric-measure spaces via optimal transport, Ann. of Math. (2) 169 (2009), 903-991.

[14] L. Molnár, Kolmogorov-Smirnov isometries and affine automorphisms of spaces of distribution functions, Cent. Eur. J. Math. 9 (2011), 789-796.

[15] L. Molnár, Lévy isometries of the space of probability distribution functions, J. Math. Anal. Appl. 380 (2011), 847-852.

[16] M.-K. von Renesse, and K.-T. Sturm, Transport inequalities, gradient estimates, entropy, and Ricci curvature, Comm. Pure Appl. Math. 58 (2005), 923-940.

[17] K.-T. Sturm, On the geometry of metric measure spaces. I, II, Acta Math. 196 (2006), 65-131 and 133-177.

[18] C. Villani, Optimal Transport: Old and New, Springer-Verlag, Berlin, 2009.

[19] C. Villani, Topics in Optimal Transportation, Graduate Studies in Mathematics, vol. 58., American Mathematical Society, Providence, RI, 2003.

[20] D. Virosztek, Maps on probability measures preserving certain distances - a survey and some new results, Acta Sci. Math. (Szeged) 84 (2018), 65-80. 
György Pál Gehér, Department of Mathematics and Statistics, University of Reading, Whiteknights, P.O. Box 220, Reading RG6 6AX, United Kingdom

Email address: gehergyuri@gmail.com or G.P.Geher@reading.ac.uk http://www.math.u-szeged.hu/ gehergy

Tamás Titkos, Alfréd Rényi Institute of Mathematics, Hungarian Academy of Sciences, ReÁltanoda u. 13-15., Budapest H-1053, Hungary, and BBS University of Applied Sciences, Alkotmány u. 9., Budapest H-1054, Hungary

Email address: titkos.tamas@renyi.mta.hu http://renyi.hu/ titkos

Dániel Virosztek, Institute of Science and Technology Austria, Am Campus 1, 3400 KlosTERNEUBURG, AUstria

Email address: daniel.virosztekeist.ac.at http://pub.ist.ac.at/ ${ }^{\text {dviroszt }}$ 